\title{
Stanniocalcin 2 alters PERK signalling and reduces cellular injury during cerulein induced pancreatitis in mice
}

\author{
Elena N Fazio ${ }^{1,2,5}$, Gabriel E DiMattia ${ }^{3,4,5,6}$, Sami A Chadi ${ }^{1,5}$, Kristin D Kernohan ${ }^{1,3,5}$ and Christopher L Pin ${ }^{1,2,5^{*}}$
}

\begin{abstract}
Background: Stanniocalcin 2 (STC2) is a secreted protein activated by (PKR)-like Endoplasmic Reticulum Kinase (PERK) signalling under conditions of ER stress in vitro. Over-expression of STC2 in mice leads to a growth-restricted phenotype; however, the physiological function for STC2 has remained elusive. Given the relationship of STC2 to PERK signalling, the objective of this study was to examine the role of STC2 in PERK signalling in vivo.

Results: Since PERK signalling has both physiological and pathological roles in the pancreas, STC2 expression was assessed in mouse pancreata before and after induction of injury using a cerulein-induced pancreatitis (CIP) model. Increased Stc2 expression was identified within four hours of initiating pancreatic injury and correlated to increased activation of PERK signalling. To determine the effect of STC2 over-expression on PERK, mice systemically expressing human STC2 (STC2 ${ }^{T g}$ ) were examined. STC2 ${ }^{T g}$ pancreatic tissue exhibited normal pancreatic morphology, but altered activation of PERK signalling, including increases in Activating Transcription Factor (ATF) 4 accumulation and autophagy. Upon induction of pancreatic injury, STC2 ${ }^{T g}$ mice exhibited limited increases in circulating amylase levels and increased maintenance of cellular junctions.
\end{abstract}

Conclusions: This study links STC2 to the pathological activation of PERK in vivo, and suggests involvement of STC2 in responding to pancreatic acinar cell injury.

\section{Background}

The stanniocalcin (STC) family of secreted glycoproteins consists of two members - STC1 and STC2 - that have $35 \%$ conserved identity and conservation of exon/intron boundaries, indicating that they are paralogs [1]. STC was first discovered in fish and shown to be involved in calcium homeostasis through effects on calcium influx $[2,3]$. Although terrestrial mammals rarely experience hypercalcemia, alterations in subcellular compartmentalization of $\mathrm{Ca}^{2+}$ are found under pathological conditions such as the accumulation of improperly folded proteins. STC2 expression is upregulated upon induction of hypoxic or endoplasmic reticulum (ER) stress in N2a neuroblastoma cells [4]. This increase in Stc2 expression was reliant on the concomitant increase of Activating Transcription Factor (ATF4) [5], a protein integral to

\footnotetext{
* Correspondence: cpin@uwo.ca

'Department of Paediatrics, The University of Western Ontario, 1151

Richmond Street, London, Ontario, N6A 3K7, Canada

Full list of author information is available at the end of the article
}

(PKR)-like endoplasmic reticulum kinase (PERK) signalling, which is part of the unfolded protein response (UPR). To date, no relationship between PERK signalling and STC2 activation has been identified in vivo.

The UPR is activated when ER homeostasis is disrupted. Alterations to proper functioning of ER machinery arise when protein load exceeds folding capacity, such as when luminal ER $\mathrm{Ca}^{2+}$ concentrations are perturbed. The UPR consists of three signalling pathways that are individually transduced by ATF6, inositolrequiring enzyme 1 (IRE1) or PERK [6,7]. Each transducer is maintained in a repressed state by binding of the ER chaperone protein glucose regulated protein 78 (GRP78/BiP) [6,7]. When unfolded proteins accumulate, they bind to GRP78/BiP resulting in its release from the UPR transducers $[8,9]$. In the case of PERK, dissociation of GRP78/BiP leads to its homodimerization and autoactivation [10]. Activation of PERK results in phosphorylation of eukaryotic translation initiation factor $2 \alpha$ (eIF2 $\alpha$ ) [11], limiting its ability to contribute to the protein
Ciomed Central

() 2011 Fazio et al; licensee BioMed Central Ltd. This is an Open Access article distributed under the terms of the Creative Commons Attribution License (http://creativecommons.org/licenses/by/2.0), which permits unrestricted use, distribution, and reproduction in any medium, provided the original work is properly cited. 
translational complex, thereby inhibiting global protein translation and alleviating the protein load on the cell [12]. Phosphorylation of eIF2 $\alpha$ also selectively upregulates translation of mRNAs from short upstream open reading frames [13]. The predominant target mRNA for peIF $2 \alpha$ is Atf4, which subsequently increases the expression of the transcriptional repressor Atf3, and growth arrest and DNA damage-inducible (Gadd) 34 [14,15]. GADD34 (alternatively known as Myd116; myeloid differentiation, primary response gene 116, or Ppp1r15a; protein phosphatase 1, regulatory [inhibitor] subunit $15 A)$ negatively regulates PERK signalling by combining with protein phosphatase 1 to dephosphorylate eIF $2 \alpha$, thereby restoring general translation $[16,17]$.

PERK is expressed in the exocrine and endocrine pancreas under normal conditions $[11,18]$ and Perk $^{-/}$mice experience deterioration of glycemic control and exocrine insufficiency over time [19]. Exocrine specific deletion of PERK revealed cellular disorganization and degranulation, increased serum amylase levels and increased cell death [20]. Our laboratory identified rapid activation of PERK signalling following induction of pancreatitis by supramaximal secretagogue stimulation and correlated decreased activation of the pathway in mice that exhibit increased sensitivity to pancreatic injury [21]. Together, these observations indicate that PERK signalling has important roles in both the physiology and pathology of the exocrine pancreas.

Given the relationship between PERK and STC2 in vitro, the objectives of this study were to (1) determine if a similar relationship exists in the exocrine pancreas and (2) gain insight into a role for STC2 as part of the UPR. Our results revealed a correlation of Stc2 expression with PERK signalling in vivo only after initiating pancreatic injury. Transgenic over-expression of STC2 in mice $\left(S T C 2^{T g}\right)$ [22] resulted in altered PERK signalling and decreased signs of acinar cell damage associated with cerulein-induced pancreatitis (CIP). These observations suggest that STC2 is linked to PERK signalling in acinar cells and may have a role in limiting damage during pancreatic injury.

\section{Results}

To determine if PERK signalling is active in pancreatic tissue, we performed IF with an antibody that recognizes only the phosphorylated form of PERK (pPERK). The accumulation of pPERK was restricted to a tight apical portion of the acinar cells (Figure 1A, green, arrows) typical of where $\mathrm{Ca}^{2+}$ is released during the process of regulated exocytosis. RT-PCR revealed no expression of Stc2 in control pancreata (Figure 1C). Similar results were obtained in control pancreata for Atf3, another downstream target of PERK signalling suggesting that PERK activation under resting conditions does not
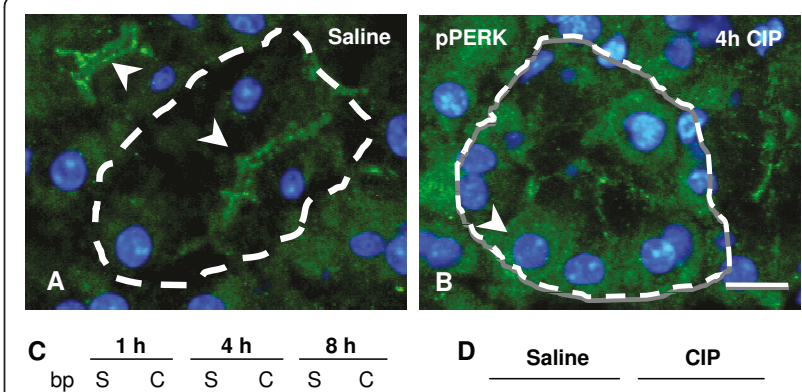

D

Saline CIP
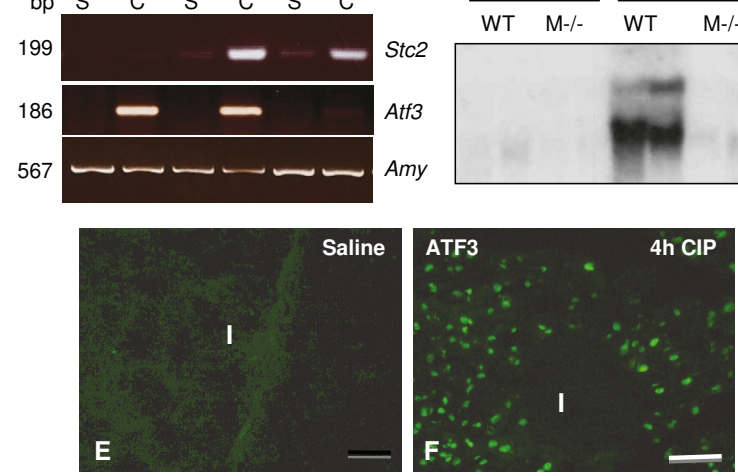

Figure 1 STC2 accumulation increases in pancreatic tissue following induction of pancreatic injury. $(A, B)$

Immunofluorescence (IF) for pPERK (green) before (A) and 4 hours after (B) initiation of CIP. pPERK (arrowhead) localizes to the apical end of acinar cells under physiological conditions and more basally during CIP. Magnification bar $=13 \mu \mathrm{M}$. DAPI was used to counter stain nuclei. (C) RT-PCR of whole pancreatic RNA extracts from saline (S) and cerulein (C) treated mice showed increased accumulation of Stc2 and Atf3 mRNA after initiation of CIP in pancreatic tissue. Amplification of Amylase (Amy) was used as a positive control for RT reactions. (D) Northern blot analysis revealed elevated Stc2 mRNA four hours after initial cerulein (CIP) or saline (S) injection. No increases in Stc2 were observed in Mist $7^{-/-}(\mathrm{M}-/-)$ pancreatic tissue under similar conditions. (E, F) IF for ATF3 expression revealed limited accumulation before CIP (E) and acinarspecific expression afterwards $(\mathbf{F})$. Magnification bar $=63 \mu \mathrm{M} . \mathrm{I}=$ islet.

activate all downstream mediators of PERK signalling. Our laboratory has shown that activation of PERK signalling increases during pancreatic injury [21]. To determine if the pathological activation of PERK leads to increased STC2 expression, pancreatic injury was initiated by cerulein-induced pancreatitis (CIP). Interestingly, CIP treatment led to a change in pPERK localization within four hours, with active PERK exhibiting a more diffuse and basally-restricted pattern of accumulation (Figure 1B, green, arrow), similar to the changes observed for acinar cell exocytosis under these conditions [23]. This change in localization was accompanied by increased Stc 2 and Atf3 mRNA accumulation (Figure 1C). Increased Stc2 expression was confirmed by Northern blot analysis four hours after induction of pancreatitis (Figure 1D, saline WT vs. CIP WT). While we were unable to detect STC2 protein by IF analysis (data not shown), increased 
accumulation of ATF3 protein was restricted to acinar cells in CIP-treated pancreatic tissue (Figure 1E, F; I = islet). To support the link between elevated Stc2 levels and injury-associated PERK activation, we examined the expression of Stc2 in Mist1 $1^{-/-}$mice, which exhibit minimal activation of PERK during injury. Mist1 is a transcription factor required for terminal differentiation of pancreatic acinar cells, and its absence in exocrine tissue results in improper activation of UPR components both normally and during injury [21]. In sharp contrast to wild type mice, Stc2 mRNA was virtually undetectable upon CIP treatment of Mist1 $1^{-/-}$mice (Figure 1D; Saline Mist1 $1^{-/-}$ vs. CIP $\left.M i s t 1^{-1}\right)$.

These findings suggest that STC2 is co-induced with PERK in acinar cells only after injury and may protect the pancreas in response to CIP. To determine if STC2 could alter the acinar cell response to pancreatic injury, we examined a mouse model in which STC2 is constitutively expressed $\left(S T C 2^{T g}\right.$; [22]). IHC confirmed increased accumulation of STC2 in $S T C 2^{T g}$ pancreata compared to wild type (WT) tissue, with STC2 accumulation in islets and exocrine tissue (Figure 2A, B). H\&E histology (Figure 2C, D) indicated that morphology was not overtly altered in $S T C 2^{T g}$ pancreatic tissue. IF staining for pancreatic differentiation markers MIST1 (exocrine, Figure 2E, F) and PDX1 (endocrine, Figure 2G, H) showed similar spatial localization and GLUT-2 accumulation was localized to the cell membrane of endocrine cells in both genotypes (Figure 2I, J). Western blot analysis confirmed equivalent levels of these markers, as well as amylase, between WT and $S T C 2^{T g}$ tissue (Figure $2 \mathrm{~K}$ ).

We next examined PERK signalling in $S T C 2^{T g}$ pancreatic tissue. PERK activation results in phosphorylation of eIF2 $\alpha$ and translation of ATF4 [24,25]. ATF4 is required for expression of Gadd34, Atf3 and Stc2 $[5,14,16]$. Western blot analysis of WT and $S T C 2^{T g}$ pancreatic tissue revealed decreased phosphorylation of both PERK and eIF2 $\alpha$ (Figure 3A, B; $\mathrm{n}=4$ animals; $\mathrm{p}<$ $0.01)$ in $S T C 2^{T g}$ tissue. Surprisingly, ATF4 accumulated to higher levels in the $S T C 2^{T g}$ pancreas, suggesting dysregulation of PERK signalling. We next examined XBP1, another mediator of the UPR that is outside of the

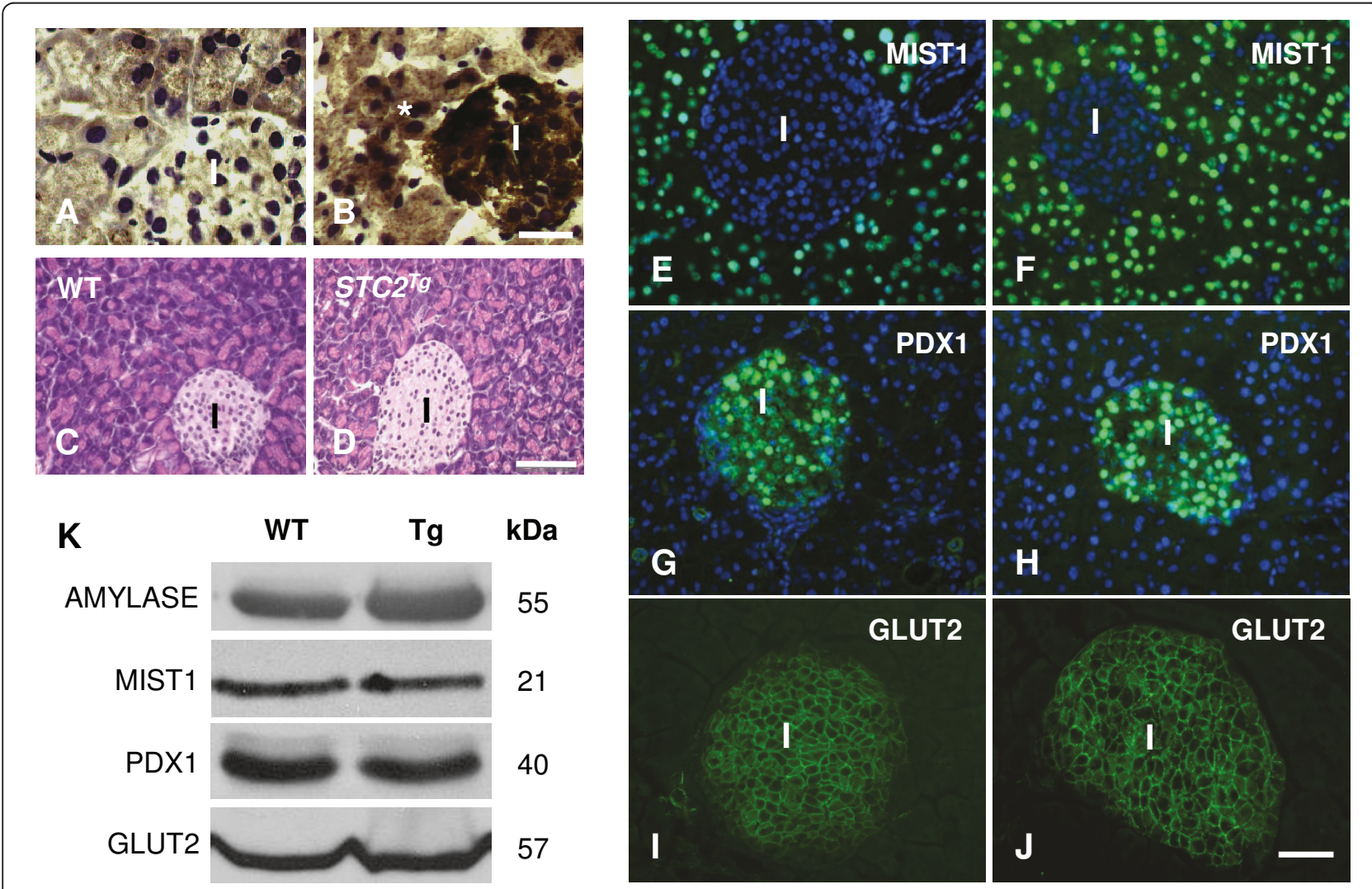

Figure 2 Overall morphology and differentiation does not appear to be affected in STC2 ${ }^{T g}$ mice. (A, B) IHC for STC2 in WT (A) and STC2 ${ }^{T g}$ (B) pancreatic islets shows that STC2 accumulates in both acinar $\left(^{*}\right)$ and islet (I) tissue only in the STC2 ${ }^{T g}$ mice. Magnification bar $=27 \mu \mathrm{m}$. Tissue was counterstained with hematoxylin to reveal tissue morphology. (C, D) Hematoxylin and eosin staining comparing general histology of pancreatic tissue in WT (C) or STC2 ${ }^{T g}$ (D) pancreata. Magnification bar $=60 \mu \mathrm{M} ;$; - islet. IF analysis for MIST1 (E, F), PDX1 (G, H) or GLUT2 (I, J) in WT $(\mathbf{E}, \mathbf{G}, \mathbf{I})$ or $\operatorname{STC2}^{T g}(\mathbf{F}, \mathbf{H}, \mathbf{J})$ pancreata. Magnification bar $=54 \mu \mathrm{m}$. (K) Representative Western blot analysis for pancreatic differentiation markers amylase, MIST1, PDX1 and GLUT2. 


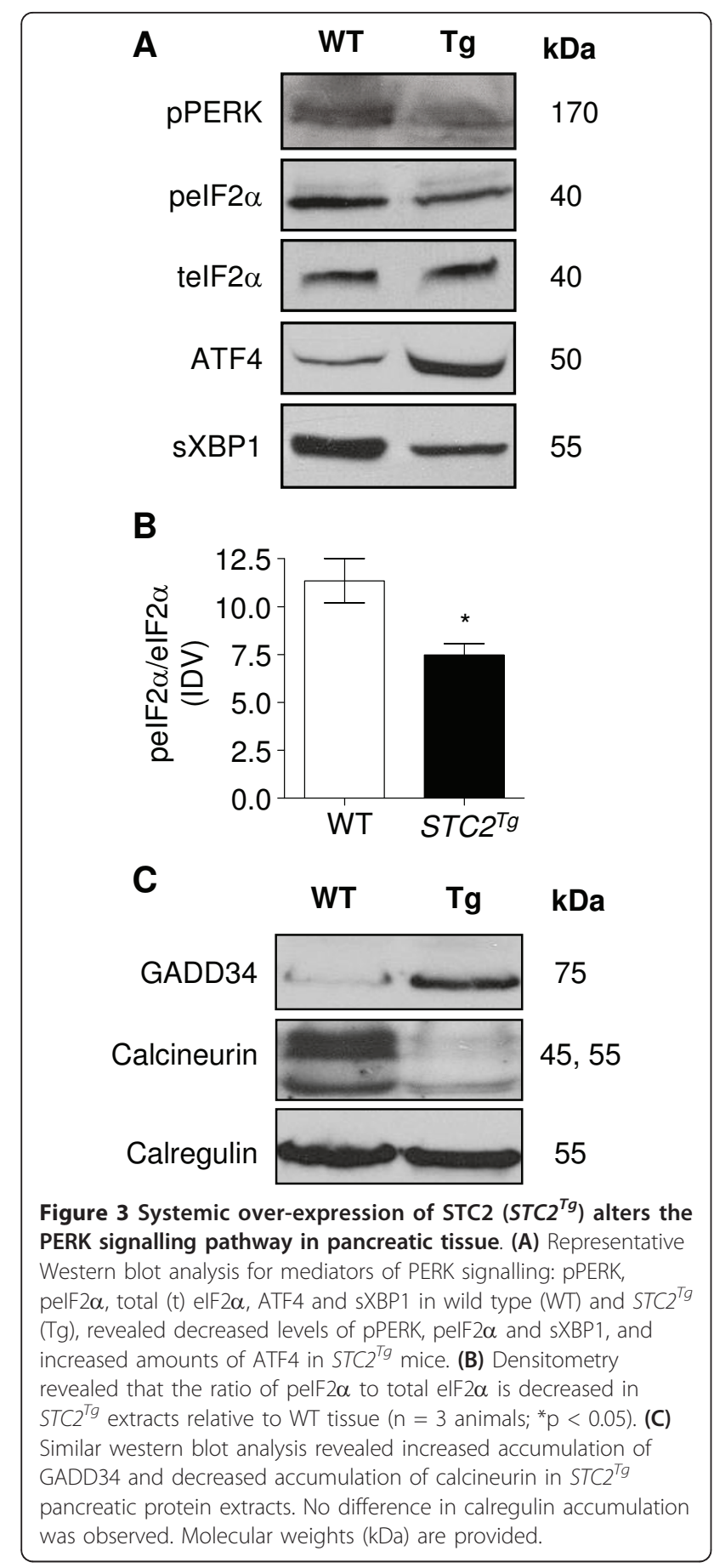

PERK signalling pathway. $X b p 1$ mRNA is targeted by the endonuclease activity of IRE1 leading to production of an active, spliced (s) form of XBP1 [26]. Western blot analysis revealed a consistent decreased in SXBP1 accumulation in $S T C 2^{T g}$ mice indicating that elevated STC2 levels may also affect the other UPR pathways.

To understand why activation of PERK and eIF $2 \alpha$ appear reduced in $\mathrm{STC} 2^{T g}$ tissue, we examined regulators of these two proteins. The phosphorylation of eIF $2 \alpha$ is reversed by GADD34 through its interaction with protein phosphatase 1 (PP1) and recent evidence indicates that calcineurin binds PERK and stimulates its autophosphorylation [27]. In $S T C 2^{T g}$ pancreatic tissue, the levels of GADD34 protein were significantly higher than wild type tissue (Figure $3 C$ ). Conversely, STC2 ${ }^{T g}$ calcineurin levels were considerably lower than WT counterparts. No changes in the ER resident protein calregulin were observed between genotypes (Figure $3 \mathrm{C}$ ). Based on these results, it appears that over-expression of STC2 affected expression of mediators and feedback mechanisms in PERK signalling.

Alterations to PERK/ATF4 signalling should have an effect on cell autophagy, a long-term response to ER stress, which is regulated by ATF4 during hypoxia [28]. Western blot analysis followed by densitometry revealed a significant increase in the cleaved form of the autophagy marker myosin associated protein 1 light chain 3 (LC3) in $S T C 2^{T g}$ pancreatic tissue (Figure $4 \mathrm{~A}, \mathrm{~B} ; \mathrm{n}=4$

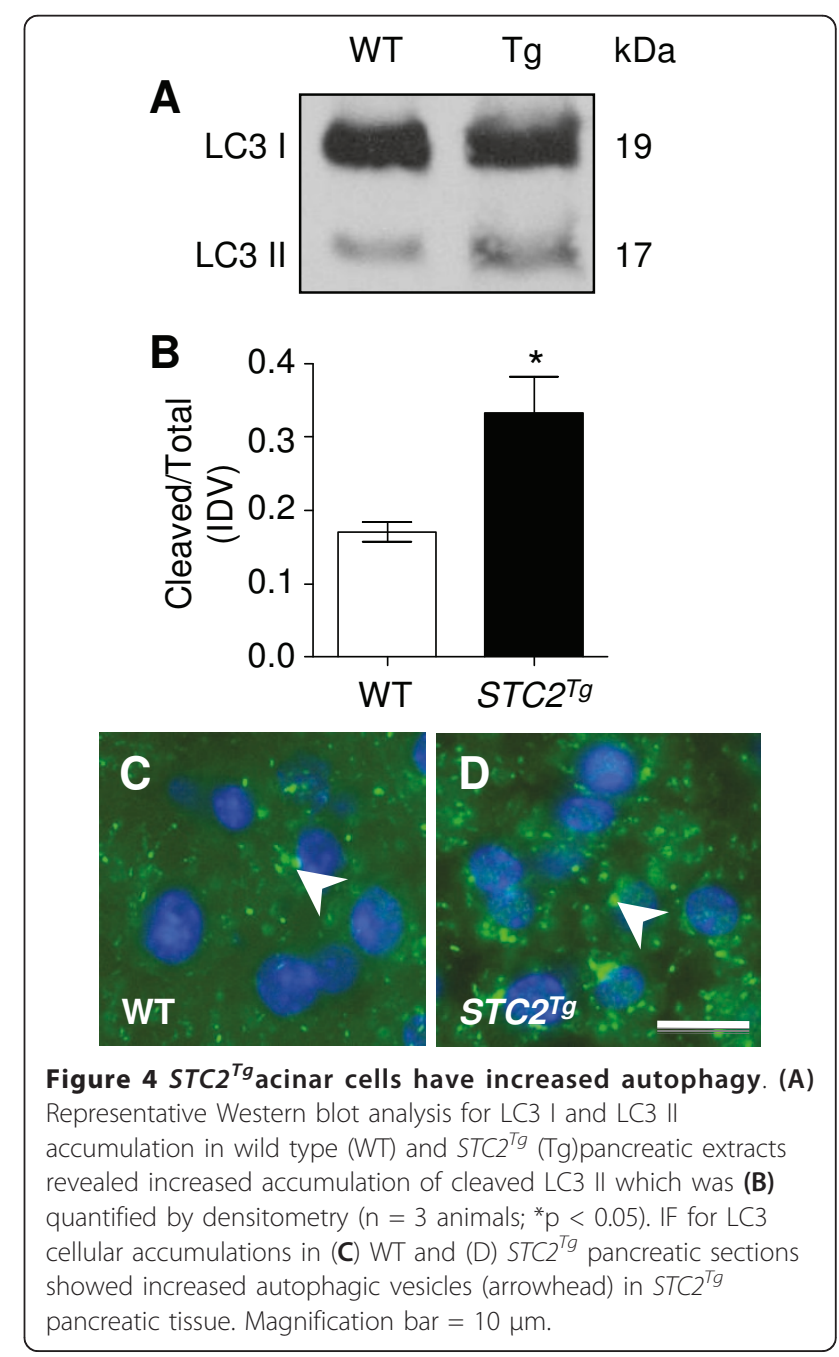


animals $\mathrm{p}<0.01$ ) consistent with increased accumulation of ATF4 in $S T C 2^{T g}$ mice. IF analysis corroborated increased autophagy as punctate LC3 staining is notably increased in $S T C 2^{T g}$ exocrine tissue (Figure 4D) when compared to WT tissue (Figure 4C).

The alterations in PERK signalling and cell autophagy suggested that $S T C 2^{T g}$ mice should have altered sensitivity to pancreatic injury. Four hours after the induction of pancreatitis, we compared circulating serum amylase levels and tissue edema between WT and $S T C 2^{T g}$ mice (Figure 5). While serum amylase levels were moderately higher in saline-treated $S T C 2^{T g}$ mice, the proportional increase in serum amylase levels following CIP was significantly lower in $S T C 2^{T g}$ mice when compared to WT mice, indicative of decreased sensitivity to pancreatic insult (Figure 5A; $\mathrm{n}=4$ animals; $\mathrm{p}<0.05$ ). However, no difference was observed in tissue edema after CIP between genotypes (Figure 5B). We next examined the effects of pancreatic injury at the cellular level by examining enzyme activation and cell structures. Western blot analysis of procarboxypeptidase (CPA) activation revealed reduced levels of active CPA in CIP $S T C 2^{T g}$ pancreatic tissue when compared to WT cerulein-treated tissue (Figure 5C, D). Interestingly, analysis of cell junction proteins showed continued expression in $S T C 2^{T g}$ tissue following CIP that was not evident in WT tissue. In WT acinar cells, the expression of $\beta$-catenin decreased to negligible levels, indicative of a loss in adherens junctions between acinar cells as expected (Figure 5E, F). However, $\beta$-catenin remained readily detectable in $S T C 2^{T g}$ pancreatic tissue after induction of CIP (Figure 5G, H). Similarly, punctate connexin 32 (Cx32) accumulation was lost in WT (Figure 5I, J) but not in $S T C 2^{T g}$ acini (Figure $5 \mathrm{~K}, \mathrm{~L}$ ) suggesting a greater disruption in gap junction complexes in WT acinar cells. Western blot analysis corroborated IF data with CIP leading to a more dramatic loss of $\beta$-catenin and Cx32 accumulation in WT extracts compared to $S T C 2^{T g}$ extracts after four hours (Figure $5 \mathrm{M}$ ) indicating that intercellular complexes were protected in $S T C 2^{T g}$ mice, suggestive of reduced cellular damage. Combined, these findings suggest that acinar cell integrity is maintained to a higher degree when acini are exposed to higher than normal levels of STC2.

As a final marker for CIP severity, we assessed the amount of acinar cell necrosis and apoptosis within the two genotypes. The ratio of necrosis to apoptosis correlates to CIP severity [29]. Visualization of apoptosis using TUNEL staining revealed increased apoptosis in both WT (Figure 6A, B; arrowheads) and $S T C 2^{T g}$ (Figure 6C, D; arrowheads) pancreatic tissue after induction of pancreatitis. Quantification of TUNEL positive cells revealed slight decreases in acinar cell apoptosis in $S T C 2^{T g}$ tissue (Figure 6E). We next examined necrosis through IF for High mobility group protein b1 (HMGB1), a protein that interacts with DNA, but upon necrotic stimulus, is released from the nucleus and the cell to participate in inflammatory responses [30,31]. Cytoplasmic staining of HMGB1 is routinely used as an indicator of cell necrosis. HMGB1 is localized to the nucleus in both WT (Figure 6F) and $S T C 2^{T g}$ (Figure $6 \mathrm{H}$ ) saline treated tissue. After induction of pancreatitis, punctate, cytoplasmic HMBG1 staining was observed in distinct patches of cells only in WT tissue (Figure 6G). All $S T C 2^{T g}$ tissue analysed was devoid of punctate cytoplasmic HMGB1 staining (Figure 6I). This analysis reveals that the levels of both apoptosis and necrosis are decreased in $S T C 2^{T g}$ tissue, indicative of less tissue damage after CIP.

\section{Discussion}

STC2 is a secreted protein that, when expressed to high levels in mice, has profound effects leading to growth restriction [22]. However, ablation of Stc2 in mice results in limited phenotypic alterations suggesting that STC2 functions may fall outside normal physiology [32]. In support of such a hypothesis, in vitro analysis has identified increased Stc2 accumulation following treatment with thapsigargin or tunicamycin, agents that activate ER stress pathways [5]. STC2 is also up-regulated in response to hypoxic insult and is expressed to higher levels in many tumor-derived cell lines [4,33]. This suggests that STC2 may be involved in responding to external challenges that activate cell stress pathways. In this study, we have identified increased Stc2 accumulation within four hours of inducing pancreatic injury that correlated with activation of the PERK signalling. Importantly, forced expression of STC2 in mice altered PERK signalling and reduced cellular damage in response to pancreatic injury. These results suggest a role for STC2 responding to and mediating the effects of cell stress in pancreatic tissue.

Studies assessing the expression of Stc2 have revealed a limited expression pattern in adult tissues. In the human pancreas, Stc2 is specifically expressed in alpha cells of the islets of Langerhans and not in acinar tissue [1]. Our mRNA analysis revealed murine pancreatic expression of Stc 2 only after induction of pancreatitis by supramaximal secretagogue stimulation. Unfortunately, IHC on CIP-treated tissue sections did not detect STC2 protein (data not shown) indicating that STC2 may be expressed at lower than detectable levels or that it is rapidly secreted into the circulation. While we have not definitively identified acini as the origin of Stc2 expression, Atf3, a downstream mediator of PERK signalling, accumulated only in acinar cells during CIP, suggesting that CIP treatment leads to a cell autonomous activation of Stc2. As observed in HEK293 and N2a neuroblastoma 

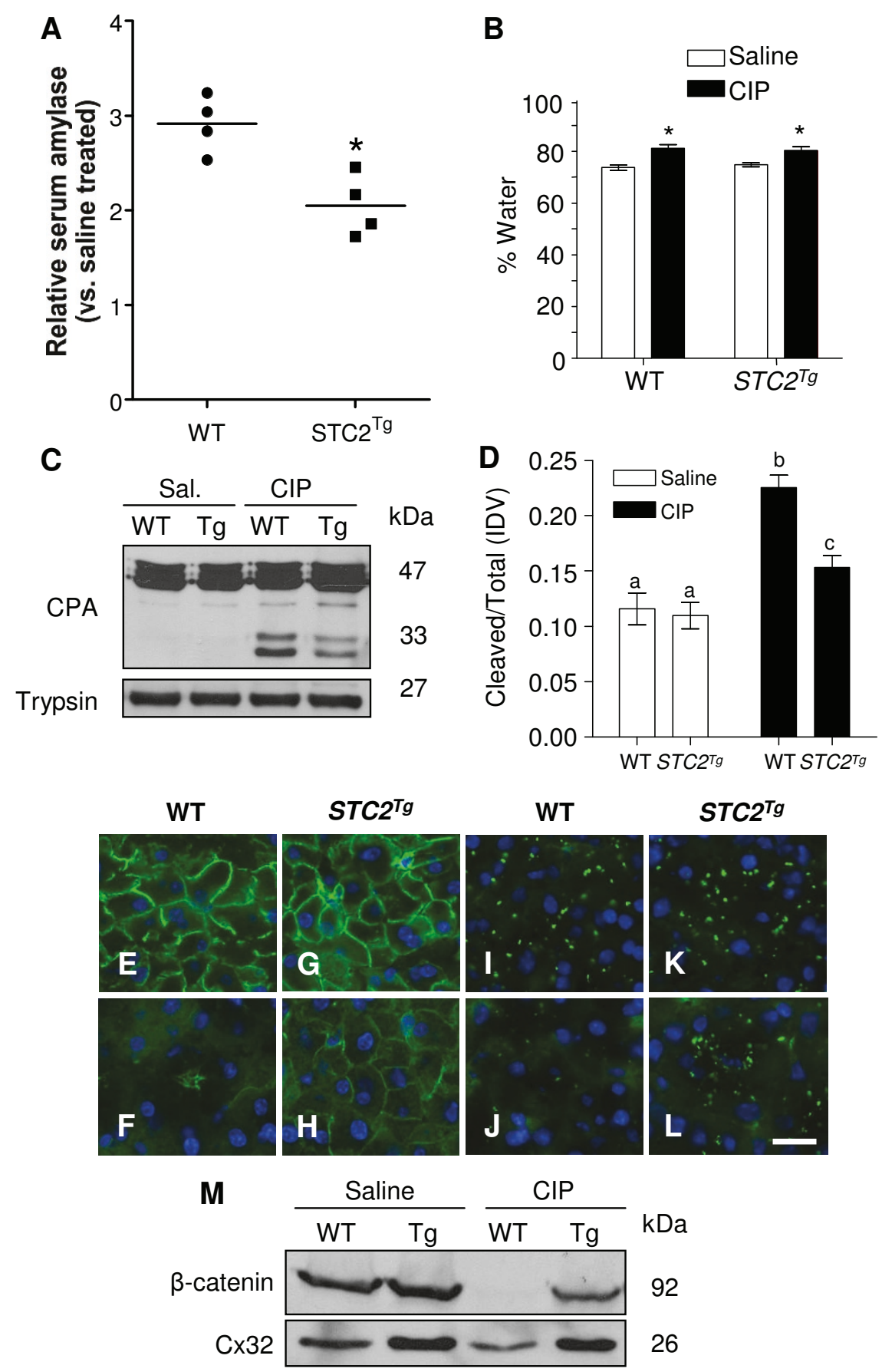

Figure 5 STC2 over-expression reduces severity of acinar cell damage during cerulein-induced pancreatic injury. (A) Analysis of serum amylase levels four hours into CIP treatment show that serum amylase levels rise approximately 3 fold in WT mice when compared to saline (•) whereas the increase in STC2 ${ }^{T g}$ mice is significantly lower at 2-fold $\left(\mathbf{m}^{*} p<0.01\right)$. (B) Tissue edema analysis revealed significant increases for both WT and STC2 ${ }^{\text {Tg }}$ tissue 4 hours into CIP (black bars) when compared to saline controls (white bars; $\mathrm{n}=4$ animals). No difference between genotypes was observed. (C) Representative Western blot for procarboxypeptidase (47 kDa) and carboxypeptidase (CPA; 33 kDa) or trypsinogen four hours after initial saline (Sal.) or cerulein (CIP) injections showed decreased accumulation of activate CPA in STC2 ${ }^{T g}$ mice four hours into CIP. Trypsinogen levels do not differ between genotypes. (D) Quantification by densitometry (graph) comparing the ratio of cleaved (active; $33 \mathrm{kDa}$ ) to total CPA revealed a significant increase in WT tissue and, to a significantly lower extent, in STC2 ${ }^{\text {Tg }}$ tissue $(n=4$ animals, letters represent statistically different values; $p<0.05)$. IF analysis for $\beta$-catenin $(\mathbf{E}-\mathbf{H})$ or Cx32 (I-L) in wild type (WT) or STC2 ${ }^{T g}(\mathrm{Tg})$ mice four hours after initial injection of saline $(\mathbf{E}, \mathbf{G}, \mathbf{I}, \mathbf{K})$ or cerulein $(\mathbf{F}, \mathbf{H}, \mathbf{J}, \mathbf{L})$ showing decreased accumulation only in WT tissue during CIP. (M) Representative Western blot analysis on pancreatic protein extracts 4 hours after initial saline or cerulein (CIP) injection confirm decreased $\beta$-catenin and $\mathrm{Cx32}$ accumulation only in WT-CIP treated tissue $(n=4)$. 
cells, the increased accumulation of Stc2 is consistent with activation of PERK signalling in response to injury [5]. Induction of Stc2 mRNA levels was not observed after CIP in Mist1 $^{-1-}$ mice, which do not activate PERK or ATF3, and experience increased severity of CIP [21]. These findings support the activation of Stc2 as a protective mechanism, potentially as a mediator of the UPR.

While previous studies have correlated increased expression of STC2 as part of the UPR, the effects of STC2 on PERK signalling have not been examined. We have now shown that systemically high levels of STC2 reduce PERK activation. $S T C 2^{T g}$ mice showed decreased phosphorylation of PERK and eIF2 $\alpha$ in pancreatic tissue suggesting that continued exposure to STC2 may lead to activation of negative feedback mechanisms. Since no change in total eIF $2 \alpha$ is observed, this difference is peIF2 $\alpha$ is specific to the phosphorylation event and not decreased eIF $2 \alpha$ accumulation. While we showed a decrease in pPERK, total PERK levels were not assessed and this could account for possible differences in activation of the pathways as well.

We have identified at least two effectors of the PERK signalling pathway that were changed in $S T C 2^{T g}$ mice. First, GADD34, which combines with protein phosphatase 1 to reduce eIF2 $\alpha$ phosphorylation [17], accumulated to higher levels. Second, we observed significantly decreased accumulation of calcineurin, which stimulates PERK autophosphorylation, thereby increasing activity [27]. Whether these changes are reflective of a direct role for STC2 in their regulation or a response to other events caused by over exposure to STC2 is unclear. However, the altered expression of these factors suggests a role for STC2 in regulating $\mathrm{Ca}^{2+}$ homeostasis since both calcineurin and PERK are affected by altered $\mathrm{Ca}^{2+}$ sequestration in the ER. These data suggest that constitutive exposure to STC2 results in depression of the activity of key factors regulating the UPR.

In light of decreased PERK and eIF2 $\alpha$ activation, it was surprising that increased accumulation of ATF4 was observed in the pancreas of $S T C 2^{T g}$ mice. Phosphorylation of eIF2 $\alpha$ is known to stimulate translation of Atf4 mRNA, leading to increased ATF4 protein accumulation. However, increased ATF4 expression, and the subsequent increase in GADD34 could be mediated by increased stability of the ATF4 protein. Recent studies have shown that ATF4 is stabilized by interaction with p300 through inhibition of ubiquitination [34], and that association with p300/CBP enhances ATF4 transcriptional ability [34,35]. It is possible that in $S T C 2^{T g}$ mice, stabilization of ATF4 is enhanced regardless of decreased phosphorylation of eIF2 $\alpha$, accounting for its increased expression. Although there is little known about non-peIF2 $\alpha$ related transcriptional activation of ATF4, increased transcriptional activation by a yet to be identified factor cannot be ruled out. 
Figure 7 summarizes a potential role for STC2 in the UPR, where over-expression of STC2 negatively affects calcineurin expression, (Figure 7, dotted line), which would lead to inhibition of calcineurin-dependent phosphorylation of PERK. Decreased phosphorylation of PERK results in decreased phosphorylation of eIF $2 \alpha$, as observed in $S T C 2^{T g}$ pancreatic tissue. Although the increased expression of ATF4 in $S T C 2^{T g}$ tissue is not intuitive considering the phosphorylation of both PERK and eIF $2 \alpha$, it reveals a potential role for STC2 in ATF4 regulation (Figure 7 , dotted arrow). This increase in ATF4 expression leads to increased GADD34 expression, which acts as a negative feedback mechanism to contribute to decreased phosphorylation of eIF2 $\alpha$ [16].

As would be expected from increased ATF4 expression, $S T C 2^{T g}$ mice also exhibited an increased level of autophagy, a downstream consequence of elevated ATF4 levels. Autophagy is important for maintenance of cellular homeostasis through balancing synthesis and degradation or recycling of cellular components. Independent studies have indicated that either phosphorylation of eIF $2 \alpha$ or expression of ATF4 is required for induction of autophagy in vitro $[28,36,37]$. The increased expression of ATF4 in $S T C 2^{T g}$ pancreatic tissue may be the cause of increased autophagy. Interestingly, we did not observe increased acinar cell apoptosis (data not

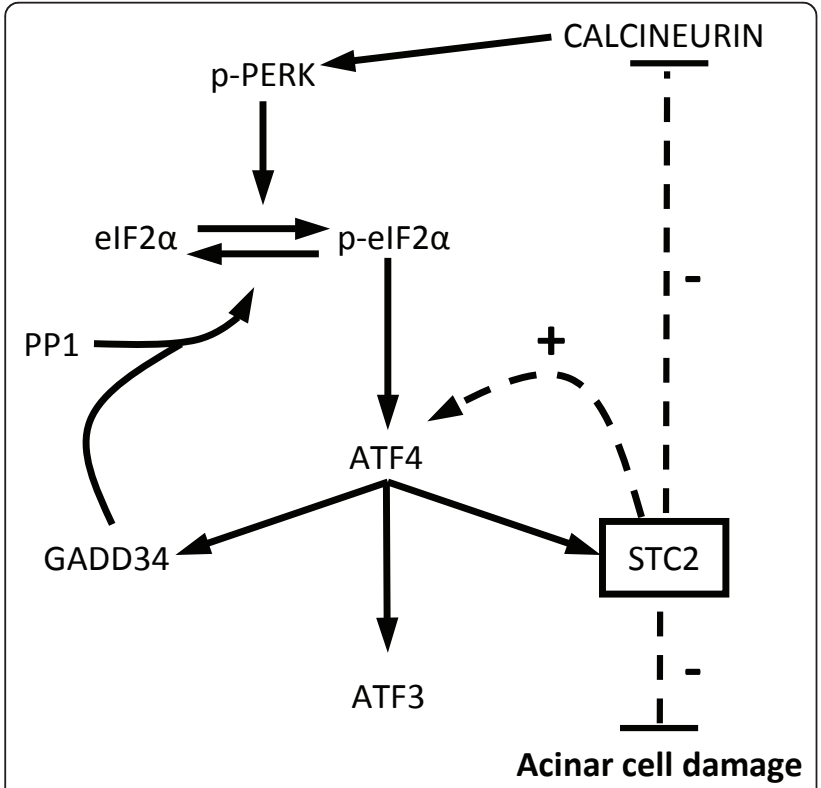

Figure 7 Schematic pathway showing a possible role for STC2 in affecting the PERK signalling pathway. The increased accumulation of ATF4 leads to increased expression of GADD34, ATF3 and STC2. Elevated levels of STC2 can alter both PERK phosphorylation and ATF4 levels through as yet undetermined mechanisms (dashed arrows), as well as promote reduced damage during pancreatic injury. Whether this protective effect of STC2 is a normal function of the protein needs to be clarified. shown), which is another downstream result of increased UPR. Therefore, it appears that only certain parts of the UPR have been activated in $S T C 2^{T g}$ mice.

Whether STC2 is protective in nature is still unclear since studies have shown it has both protective [38] and detrimental roles $[4,33]$ in cancer progression in vitro. Our results suggest a protective advantage following exocrine pancreatic injury in vivo. STC2 ${ }^{T g}$ mice exhibit a decreased proportional increase in serum amylase levels, increased maintenance of cellular junctions, decreased apoptosis and necrosis and decreased activation of CPA in exocrine pancreatic tissue, all suggestive of decreased pancreatitis severity. In addition, the enhanced autophagy observed in $S T C 2^{T g}$ acinar tissue would facilitate more rapid degradation of resident digestive enzymes upon injury, thereby limiting damage to cellular contents. This would account for increased maintenance of cellular junctions. However, to truly understand the role of STC2 in pancreatic injury, further experiments in mice lacking Stc2 mice should be performed.

\section{Conclusions}

In conclusion, this is the first study to correlate PERK signalling and STC2 in vivo, and suggests two novel roles for STC2, as a downstream effector of PERK signalling and possible protective factor during pancreatic injury. This is also the first time that STC2 has been shown to affect proteins that regulate $\mathrm{Ca}^{2+}$-mediated response in the cell.

\section{Methods \\ Mouse handling}

For characterization of Stc2 expression during ceruleininduced pancreatitis (CIP), wild type and Mist $^{-1 /}$ mice were maintained on a C57/Bl6 background. For analysis of pancreatitis severity, $S T C 2^{T g}$ and wild type mice are maintained on a C57/Bl6 $\times$ CBA background. Mice carrying a targeted ablation of the Mist1 gene (Mist $1^{-/-}$) [39] and transgenic mice that express STC2 $\left(S T C 2^{T g}\right)$ from the $C M V$ promoter + chicken $\beta$-actin promoter [22], have previously been described. All experiments were approved by the Animal Care Committee at the University of Western Ontario (Protocol \#116-2008) and mice handled according to regulations stipulated by the Canadian Council on Animal Care.

\section{Induction of pancreatitis}

To induce pancreatic injury, 2-4 month-old female mice were given up to seven hourly intraperitoneal injections of cerulein ( $50 \mu \mathrm{g} / \mathrm{kg}$ body weight; Sigma-Aldrich). As a control, mice were injected with $0.9 \%$ saline. Mice were sacrificed 1, 4 or 8 hours after initial cerulein injection and pancreatic tissue from each was immediately 
processed to isolate RNA, protein, and tissue sections for histological or immunohistochemical analysis. To assess serum amylase, blood was obtained via cardiac puncture, placed on ice for 20 minutes and centrifuged at $4^{\circ} \mathrm{C}, 2500 \mathrm{rpm}$ for 15 minutes. Serum amylase was quantified using a Phadebas amylase assay (Magle Life Sciences, Lund, Sweden) as per manufacturer's instructions.

\section{Tissue preparation and histology}

Pancreatic tissue was either directly embedded in OCT or incubated in formalin for 24 hours followed by paraffin embedding. For morphological analysis, paraffin embedded tissue was sectioned to $6 \mu \mathrm{M}$ and stained with hematoxylin and eosin. Immunofluorescent (IF) analysis was performed on fresh frozen sections as described previously [40]. Primary antibodies and their dilutions are listed in Table 1. Following primary antibody incubation and PBS washes, sections were incubated for 1 hour with the secondary antibodies of either anti-rabbit FITC or anti-mouse TRITC (1:250; SigmaAldrich). After further washing, sections were incubated with 4',6-diamidino-2-phenylindole (DAPI; 1:1000) and slides were mounted with Vectashield (Vector Laboratories, Burlingame CA, USA). Histological staining was visualized with a Leica DMIOS upright microscope and images were captured using OpenLab 4.0.3 Software (PerkinElmer, Waltham, MA, USA).

\section{TUNEL analysis}

TUNEL analysis was used to identify apoptotic cells using the In situ Cell Death Detection kit (Roche, Laval, QC, Canada) following manufacturer's instructions.
Briefly, frozen sections were fixed, washed and permeabilized before labelling with TUNEL reaction mixture for 60 minutes. After washing, sections were counterstained with DAPI, coverslipped and visualized using a Leica DMIOS upright microscope and images were captured using OpenLab 4.0.3 Software (PerkinElmer, Waltham, MA, USA). Analysis of percent apoptotic cells was carried out by comparing TUNEL positive cells to total cell number. Cell counts based on DAPI were done using Image J software, and TUNEL positive cells were determined manually. Ten random fields of view from 4 sections per animal were used for analysis.

\section{RNA isolation, Northern blotting and Real-Time RT-PCR}

RNA was isolated from the splenic portion of the pancreas and processed using TRIzol (Invitrogen, Burlington, ON, Canada) as described [21]. Northern blotting was performed as previously described [39]. Thirty $\mu \mathrm{g}$ of RNA was resolved by electrophoresis on a $1 \%$ agarose/formaldehyde gel and blotted onto Hybond membrane (GE Healthcare, Baie d'Urfe, QC, Canada). Membranes were hybridized overnight at $42^{\circ} \mathrm{C}$ with an $\alpha-{ }^{32} \mathrm{PdCTP}$ radiolabelled probe for Stc 2 or for $18 S$ rRNA as a loading control.

For RT-PCR, two $\mu \mathrm{g}$ of RNA was reverse transcribed using Improm-II reverse transcriptase and random primers (Promega, Madison WI, USA). RT-PCR for Stc2, Atf3, amylase and $\beta$-actin was performed with $1 \mu \mathrm{L}$ of cDNA and Taq DNA polymerase (Promega, Madison WI, USA).

\section{Protein isolation and immunoblot analysis}

Protein extraction was carried out to obtain both nuclear and cytoplasmic fractions. Briefly, 5 volumes of

Table 1 List of Primary Antibodies Used in Fazio et al (2011)

\begin{tabular}{|c|c|c|c|c|c|}
\hline Antigen & Species & Company & Dilution & Method & Reference \\
\hline ATF3 & Rabbit & Santa Cruz Biotechnology (Santa Cruz, CA, USA) & $1: 1000$ & $\mathrm{IB} / \mathrm{IF}$ & Wu et al. (2010). Nature. 465: 368-372 \\
\hline ATF4 & Rabbit & Santa Cruz Biotechnology & $1: 500$ & $\mathrm{IB}$ & Matsushita et al. (2009). Mol. Cell. Biol. 29: 5843-5857. \\
\hline Calcineurin & Mouse & Sigma (St. Louis, MO, USA) & $1: 1000$ & $\mathrm{IB}$ & \\
\hline Calregulin & Rabbit & Santa Cruz Biotechnology & $1: 1000$ & $\mathrm{IB}$ & \\
\hline Pro-CPA & Rabbit & AbD Serotec (Oxford, UK) & $1: 2000$ & IB & \\
\hline$\beta$-catenin & Mouse & Transduction Laboratories (San Jose, CA, USA) & $1: 1000$ & $\mathrm{IB} / \mathrm{IF}$ & \\
\hline Connexin 32 & Mouse & Millipore (Temecula, CA, USA) & $1: 1000$ & $\mathrm{IB} / \mathrm{IF}$ & \\
\hline pelF $2 \alpha$ & Rabbit & Invitrogen (Carlsbad, CA, USA) & $1: 1000$ & $\mathrm{IB}$ & Harding et al. (2009). PNAS. 106(6), 1832-1837 \\
\hline total elF2 $\alpha$ & Rabbit & Cell Signalling (Beverly, MA, USA) & $1: 1000$ & $\mathrm{IB}$ & Nanbo et al. (2002) EMBO J. 21, 954-965. \\
\hline GADD34 & Rabbit & Santa Cruz Biotechnology & $1: 500$ & $\mathrm{IB}$ & Makris et al. (2000). Mol. Cell. 5: 969-979. \\
\hline GLUT2 & Rabbit & Millipore & $1: 1000$ & $\mathrm{IB} / \mathrm{IF}$ & \\
\hline HMGB1 & Rabbit & Novus Biologicals (Littleton, CO, USA) & $1: 500$ & IF & \\
\hline LC3 & Rabbit & Novus Biologicals & $1: 1000$ & $\mathrm{IB} / \mathrm{IF}$ & \\
\hline MIST1 & Rabbit & ProSci Incorporated (Poway, CA, USA) & $1: 500$ & $\mathrm{IB} / \mathrm{IF}$ & \\
\hline PDX1 & Rabbit & Abcam (Cambridge, MA, USA) & 1:1000 & $\mathrm{IB} / \mathrm{IF}$ & \\
\hline PPERK & Rabbit & Santa Cruz Biotechnology & 1:500 & $\mathrm{IB} / \mathrm{IF}$ & Denis et al. (2010). Neuroscience. 170(4):1035-44 \\
\hline Trypsin & Rabbit & Chemicon (Temecula, CA, USA) & $1: 2000$ & $\mathrm{IB}$ & \\
\hline
\end{tabular}

1 - Abbreviations; IB, immunoblotting; IF, Immunofluorescence. 
buffer CE pH 7.6 (10 mm Hepes, $60 \mathrm{mM} \mathrm{KCl,} 1 \mathrm{mM}$ EDTA, $0.075 \% \mathrm{v} / \mathrm{v}$ NP-40, $1 \mathrm{mM}$ DTT, $1 \mathrm{mM}$ PMSF) were added to whole pancreatic tissue prior to homogenization. Homogenized cells were centrifuged at 1000 $\mathrm{rpm}, 4^{\circ} \mathrm{C}$ for 4 minutes. The supernatant was removed and saved as cytoplasmic extract. The nuclear pellet was gently washed with CE buffer lacking NP-40 and centrifuged again. After removal of the supernatant, the nuclear pellet was covered with 1 pellet volume of NE buffer (20 $\mathrm{mm}$ Tris- $\mathrm{HCl}, 420 \mathrm{mM} \mathrm{NaCl}, 1.5 \mathrm{~mm} \mathrm{MgCl}_{2}, 0.2 \mathrm{mM}$ EDTA, $1 \mathrm{mM}$ PMSF, 25\% w/v glycerol) and the $\mathrm{NaCl}$ concentration was adjusted to $400 \mathrm{mM}$ for the entire volume. An additional pellet volume of NE buffer was added followed by vortexing for resuspension. The extract was incubated on ice for 10 minutes with intermittent vortexing. Both cytoplasmic and nuclear extracts were centrifuged at $14,000 \mathrm{rpm}, 4^{\circ} \mathrm{C}$, for 10 minutes to pellet any debris. Nuclear extracts were used for analysis of MIST1, PDX1, XBP1 and ATF4 levels, whereas cytoplasmic extracts were used for all other immunoblot analysis.

Electrophoresis and immunoblotting were carried out as previously described [41]. For immunoblot analysis, 40 to $60 \mu \mathrm{g}$ of pancreatic protein was resolved by SDS/ PAGE and transferred to PVDF membrane (Biorad, Mississauga, ON, Canada). Blots were blocked with 5\% nonfat dry milk (NFDM) and probed with primary antibodies diluted in $5 \%$ NFDM overnight at $4{ }^{\circ} \mathrm{C}$. For primary antibodies and their dilutions, see Table 1 . Blots were washed three times in PBS supplemented with Tween20 (PBS-T) then incubated with anti-rabbit or antimouse horseradish peroxidase conjugated secondary antibody (1:10,000 or 1:2000, Jackson Labs, Bar Harbor, ME, USA) in 5\% NFDM for one hour. Blots were subjected to another series of washes in PBS- $T$, incubated with Western Lightning chemiluminescence substrate (Perkin Elmer, Waltham MA, USA), exposed to X-ray film (Fisher Scientific, Ottawa, ON, Canada) and developed. Denistometry was performed on immunoblot autoradiographs by analysis with a FluorChem 8800 documentation system and accompanying FluorChem 8800 software (Alpha Innotech, San Leandro CA, USA).

\section{Statistical Analyses}

All statistical analyses were performed using Graphpad Prism 4.02 (Graphpad Software, San Diego CA, USA). Carboxypeptidase cleavage and serum amylase levels were analyzed using a two-way ANOVA followed by a Bonferroni post-hoc test. Levels of phosphorylated eIF2 $\alpha$ and LC3 cleavage were statistically analyzed using a Mann-Whitney test.

\section{Acknowledgements}

The authors would like to thank members of the Pin and DiMattia laboratories, especially Charis Johnson, Christin Giller and Yudith Ramos-
Valdes for their support and suggestions in these studies. This work was funded by operating grants from the Canadian Institutes of Health Research (CP - MOP 53083; GD - MOP 53128). C.P. is supported by a salary award from the CIHR. E.F. is supported by a doctoral research award from the $\mathrm{CIHR} / \mathrm{CDHF}$

\section{Author details}

${ }^{1}$ Department of Paediatrics, The University of Western Ontario, 1151 Richmond Street, London, Ontario, N6A 3K7, Canada. ${ }^{2}$ Department of Physiology and Pharmacology, The University of Western Ontario, 1151 Richmond St., London, Ontario, N6A 3K7, Canada. ${ }^{3}$ Department of Biochemistry, The University of Western Ontario, 1151 Richmond St., London, Ontario, N6A 3K7, Canada. ${ }^{4}$ Department of Oncology, The University of Western Ontario, 1151 Richmond St., London, Ontario, N6A 3K7, Canada. ${ }^{5}$ Children's Health Research Institute, 800 Commissioners Rd. E, London, Ontario, N6C 2V5, Canada. ' London Regional Cancer Program, 790 Commissioners Rd. E., London, Ontario, N6C 2V5, Canada.

\section{Authors' contributions}

EF carried out the animal studies, protein and gene expression analysis, performed the statistical analysis and drafted the manuscript. KK carried out some immunofluorescent analysis and SC performed Northern blot analysis. GD provided mouse lines and participated in its design and coordination and helped draft the manuscript. CP conceived the study, and participated in its design and coordination and helped to draft the manuscript. All authors read and approved the final manuscript.

Received: 20 January 2011 Accepted: 5 May 2011 Published: 5 May 2011

\section{References}

1. Moore EE, Kuestner RE, Conklin DC, Whitmore TE, Downey W, Buddle MM, Adams RL, Bell LA, Thompson DL, Wolf A, Chen L, Stamm MR, Grant FJ, Lok S, Ren H, De Jongh KS: Stanniocalcin 2: characterization of the protein and its localization to human pancreatic alpha cells. Horm Metab Res 1999, 31(7):406-414.

2. Flik G, Labedz T, Neelissen JA, Hanssen RG, Wendelaar Bonga SE, Pang PK: Rainbow trout corpuscles of Stannius: stanniocalcin synthesis in vitro. The American journal of physiology 1990, 258(5 Pt 2):R1157-1164.

3. Sundell $\mathrm{K}$, Bjornsson BT, Itoh H, Kawauchi H: Chum salmon (Oncorhynchus keta) stanniocalcin inhibits in vitro intestinal calcium uptake in Atlantic cod (Gadus morhua). J Comp Physiol B 1992, 162(6):489-495.

4. Law AY, Wong CK: Stanniocalcin-2 is a HIF-1 target gene that promotes cell proliferation in hypoxia. Exp Cell Res 2009, 316(3):466-476.

5. Ito D, Walker JR, Thompson CS, Moroz I, Lin W, Veselits ML, Hakim AM, Fienberg AA, Thinakaran G: Characterization of stanniocalcin 2, a novel target of the mammalian unfolded protein response with cytoprotective properties. Molecular and cellular biology 2004, 24(21):9456-9469.

6. Bertolotti A, Zhang Y, Hendershot LM, Harding HP, Ron D: Dynamic interaction of BiP and ER stress transducers in the unfolded-protein response. Nat Cell Biol 2000, 2(6):326-332.

7. Okamura K, Kimata Y, Higashio H, Tsuru A, Kohno K: Dissociation of Kar2p/ BiP from an ER sensory molecule, Ire1p, triggers the unfolded protein response in yeast. Biochemical and biophysical research communications 2000, 279(2):445-450.

8. Freiden PJ, Gaut JR, Hendershot LM: Interconversion of three differentially modified and assembled forms of BiP. Embo J 1992, 11(1):63-70.

9. Laitusis AL, Brostrom MA, Brostrom CO: The dynamic role of GRP78/BiP in the coordination of mRNA translation with protein processing. The Journal of biological chemistry 1999, 274(1):486-493.

10. Liu CY, Kaufman RJ: The unfolded protein response. J Cell Sci 2003, 116(Pt 10):1861-1862.

11. Shi Y, Vattem KM, Sood R, An J, Liang J, Stramm L, Wek RC: Identification and characterization of pancreatic eukaryotic initiation factor 2 alphasubunit kinase, PEK, involved in translational control. Molecular and cellular biology 1998, 18(12):7499-7509.

12. Harding HP, Zhang $Y$, Ron D: Protein translation and folding are coupled by an endoplasmic-reticulum-resident kinase. Nature 1999, 397(6716):271-274.

13. Hinnebusch AG: Translational regulation of yeast GCN4. A window on factors that control initiator-trna binding to the ribosome. The Journal of biological chemistry 1997, 272(35):21661-21664. 
14. Jiang HY, Wek SA, McGrath BC, Lu D, Hai T, Harding HP, Wang X, Ron D, Cavener DR, Wek RC: Activating transcription factor 3 is integral to the eukaryotic initiation factor 2 kinase stress response. Molecular and cellular biology 2004, 24(3):1365-1377.

15. Ma K, Vattem KM, Wek RC: Dimerization and release of molecular chaperone inhibition facilitate activation of eukaryotic initiation factor- 2 kinase in response to endoplasmic reticulum stress. The Journal of biological chemistry 2002, 277(21):18728-18735.

16. Ma $Y$, Hendershot LM: Delineation of a negative feedback regulatory loop that controls protein translation during endoplasmic reticulum stress. The Journal of biological chemistry 2003, 278(37):34864-34873.

17. Novoa I, Zeng H, Harding HP, Ron D: Feedback inhibition of the unfolded protein response by GADD34-mediated dephosphorylation of elF2alpha. J Cell Biol 2001, 153(5):1011-1022.

18. Sood R, Porter AC, Ma K, Quilliam LA, Wek RC: Pancreatic eukaryotic initiation factor-2alpha kinase (PEK) homologues in humans, Drosophila melanogaster and Caenorhabditis elegans that mediate translational control in response to endoplasmic reticulum stress. Biochem J 2000, 346(Pt 2):281-293.

19. Harding HP, Zeng $H$, Zhang $Y$, Jungries $R$, Chung $P$, Plesken $H$, Sabatini DD, Ron D: Diabetes mellitus and exocrine pancreatic dysfunction in perk-/mice reveals a role for translational control in secretory cell survival. $\mathrm{Mol}$ Cell 2001, 7(6):1153-1163.

20. lida $K$, Li Y, McGrath BC, Frank A, Cavener DR: PERK elF2 alpha kinase is required to regulate the viability of the exocrine pancreas in mice. $B M C$ Cell Biol 2007, 8:38

21. Kowalik AS, Johnson CL, Chadi SA, Weston JY, Fazio EN, Pin CL: Mice lacking the transcription factor Mist1 exhibit an altered stress response and increased sensitivity to caerulein-induced pancreatitis. American journal of physiology 2007, 292(4):G1123-1132.

22. Gagliardi AD, Kuo EY, Raulic S, Wagner GF, DiMattia GE: Human stanniocalcin-2 exhibits potent growth-suppressive properties in transgenic mice independently of growth hormone and IGFs. Am $J$ Physiol Endocrinol Metab 2005, 288(1):E92-105.

23. Cosen-Binker LI, Lam PP, Binker MG, Gaisano HY: Alcohol-induced protein kinase Calpha phosphorylation of Munc18c in carbachol-stimulated acini causes basolateral exocytosis. Gastroenterology 2007, 132(4):1527-1545.

24. Vattem KM, Wek RC: Reinitiation involving upstream ORFs regulates ATF4 mRNA translation in mammalian cells. Proc Natl Acad Sci USA 2004, 101(31):11269-11274

25. Lu PD, Harding HP, Ron D: Translation reinitiation at alternative open reading frames regulates gene expression in an integrated stress response. J Cell Biol 2004, 167(1):27-33.

26. Calfon M, Zeng H, Urano F, Till JH, Hubbard SR, Harding HP, Clark SG, Ron D: IRE1 couples endoplasmic reticulum load to secretory capacity by processing the XBP-1 mRNA. Nature 2002, 415(6867):92-96.

27. Bollo M, Paredes RM, Holstein D, Zheleznova N, Camacho P, Lechleiter JD: Calcineurin interacts with PERK and dephosphorylates calnexin to relieve ER stress in mammals and frogs. PLoS One 2010, 5(8):e11925.

28. Rzymski T, Milani M, Pike L, Buffa F, Mellor HR, Winchester L, Pires I, Hammond E, Ragoussis I, Harris AL: Regulation of autophagy by ATF4 in response to severe hypoxia. Oncogene 2010, 29(31):4424-4435.

29. Bhatia M: Apoptosis versus necrosis in acute pancreatitis. American journal of physiology 2004, 286(2):G189-196.

30. Fortunato F, Burgers $H$, Bergmann F, Rieger P, Buchler MW, Kroemer G, Werner J: Impaired autolysosome formation correlates with Lamp-2 depletion: role of apoptosis, autophagy, and necrosis in pancreatitis. Gastroenterology 2009, 137(1):350-360, 360 e351-355.

31. Scaffidi P, Misteli T, Bianchi ME: Release of chromatin protein HMGB1 by necrotic cells triggers inflammation. Nature 2002, 418(6894):191-195.

32. Chang AC, Hook J, Lemckert FA, McDonald MM, Nguyen MA, Hardeman EC, Little DG, Gunning PW, Reddel RR: The murine stanniocalcin 2 gene is a negative regulator of postnatal growth. Endocrinology 2008, 149(5):2403-2410.

33. Law AY, Wong CK: Stanniocalcin-2 promotes epithelial-mesenchymal transition and invasiveness in hypoxic human ovarian cancer cells. Exp Cell Res 2010, 316(20):3425-3434.

34. Lassot I, Estrabaud E, Emiliani S, Benkirane M, Benarous R, MargottinGoguet F: p300 modulates ATF4 stability and transcriptional activity independently of its acetyltransferase domain. The Journal of biological chemistry 2005, 280(50):41537-41545.
35. Cherasse Y, Maurin AC, Chaveroux C, Jousse C, Carraro V, Parry L, Deval C, Chambon C, Fafournoux P, Bruhat A: The p300/CBP-associated factor (PCAF) is a cofactor of ATF4 for amino acid-regulated transcription of CHOP. Nucleic Acids Res 2007, 35(17):5954-5965.

36. Kouroku Y, Fujita E, Tanida I, Ueno T, Isoai A, Kumagai H, Ogawa S, Kaufman RJ, Kominami E, Momoi T: ER stress (PERK/elF2alpha phosphorylation) mediates the polyglutamine-induced LC3 conversion, an essential step for autophagy formation. Cell Death Differ 2007, 14(2):230-239

37. Milani M, Rzymski T, Mellor HR, Pike L, Bottini A, Generali D, Harris AL: The role of ATF4 stabilization and autophagy in resistance of breast cancer cells treated with Bortezomib. Cancer Res 2009, 69(10):4415-4423.

38. Raulic S, Ramos-Valdes Y, DiMattia GE: Stanniocalcin 2 expression is regulated by hormone signalling and negatively affects breast cancer cell viability in vitro. J Endocrinol 2008, 197(3):517-529.

39. Pin CL, Rukstalis JM, Johnson C, Konieczny SF: The bHLH transcription factor Mist1 is required to maintain exocrine pancreas cell organization and acinar cell identity. J Cell Biol 2001, 155(4):519-530.

40. Fazio EN, Pin CL: Mist1-null mice are resistant to streptozotocin-induced beta cell damage. Biochemical and biophysical research communications 2007, 353(3):823-828

41. Johnson CL, Kowalik AS, Rajakumar N, Pin CL: Mist1 is necessary for the establishment of granule organization in serous exocrine cells of the gastrointestinal tract. Mech Dev 2004, 121(3):261-272.

doi:10.1186/1471-2121-12-17

Cite this article as: Fazio et al:: Stanniocalcin 2 alters PERK signalling and reduces cellular injury during cerulein induced pancreatitis in mice. BMC Cell Biology 2011 12:17.

\section{Submit your next manuscript to BioMed Central and take full advantage of:}

- Convenient online submission

- Thorough peer review

- No space constraints or color figure charges

- Immediate publication on acceptance

- Inclusion in PubMed, CAS, Scopus and Google Scholar

- Research which is freely available for redistribution

Submit your manuscript at www.biomedcentral.com/submit
C Biomed Central 